\title{
Phenotypic characterisation of
}

\section{T-lymphocytes in COPD: abnormal \\ CD4+CD25+ regulatory T-lymphocyte}

\section{response to tobacco smoking}

\author{
B. Barceló*,\#, J. Pons ${ }^{\#, \uparrow, ~ J . M . ~ F e r r e r ~}{ }^{\#, \oplus, ~ J . ~ S a u l e d a ~}{ }^{\#,+, \S}$, \\ A. Fuster ${ }^{+}$and A.G.N. Agustí, ${ }^{\#,+, \S}$
}

ABSTRACT: Tobacco smoking induces an inflammatory response in the lungs of all smokers but, for reasons that are still poorly understood, only a proportion of them develop chronic obstructive pulmonary disease (COPD). Recent evidence indicates that this inflammatory response persists after smoking cessation, suggesting some type of auto-perpetuation mechanism similar to that described in autoimmune disorders. T-lymphocytes (CD4+ and CD8+) have been implicated in the pathogenesis of both COPD and several autoimmune processes. A subtype of regulatory CD4+ T-cells expressing CD25 (Tregs) plays a critical role in the maintenance of peripheral tolerance and the prevention of autoimmunity, but their potential role in COPD has not been explored. The present study sought to evaluate maturation (CD45RA/CD45R0) and activation markers (CD28) of T-lymphocytes and to explore potential Treg abnormalities in COPD.

Flow cytometry was used to characterise T-lymphocytes obtained from blood and bronchoalveolar lavage fluid (BALF) in 23 patients with moderate COPD, 29 smokers with normal lung function and seven never-smokers.

The main findings were that in BALF: patients with COPD showed higher CD8+CD45RA+ and lower CD8+CD45R0+ than smokers with normal lung function; and compared with never-smokers, smokers with preserved lung function showed a prominent upregulation of Tregs that was absent in patients with COPD.

These observations indicate a final maturation-activation state of CD8+ T-lymphocytes in chronic obstructive pulmonary disease and, for the first time, identify a blunted regulatory T-cell response to tobacco smoking in these patients, further supporting a potential involvement of the acquired immune response in the pathogenesis of the disease.

KEYWORDS: Flow cytometry, lung inflammation, mucosal homeostasis, tissue repair

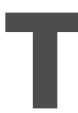

obacco smoking is the main risk factor for chronic obstructive pulmonary disease (COPD) [1]. It causes an inflammatory response in all smokers but, for reasons that are still poorly understood, only a proportion of them develop COPD $[2,3]$. It is likely that the intensity and/or type of inflammation elicited by smoking differs between smokers who manage to preserve lung function despite their habit and those who eventually develop COPD.

Several cell lines, including macrophages, neutrophils and lymphocytes, participate in the inflammatory response that characterises COPD

For editorial comments see page 486 .
[4]. In order to understand why only some smokers develop the disease, it is imperative to describe the phenotypic characteristics of these different cell types in patients with COPD compared with those determined in smokers with normal lung function and never-smokers. Following this research strategy, the current authors' group has recently reported qualitative and quantitative differences in alveolar macrophages [5] and $\gamma \delta$ T-lymphocytes [6] in smokers with and without COPD and never-smokers. However, little is known about cytotoxic and memory T-cells.

Conversely, the concept that COPD might have an autoimmune component has gained increased
AFFILIATIONS

Depts of ${ }^{*}$ Clinical Analysis, and -Immunology, and

+Pneumology, Hospital Universitari Son Dureta, Palma de Mallorca, and

§Fundació Caubet-CIMERA, and

${ }^{\#}$ Ciber Enfermedades Respiratorias, Mallorca, Spain.

CORRESPONDENCE

A.G.N. Agustí

Servei de Pneumologia

Hospital Universitari Son Dureta

Andrea Doria 55

07014-Palma de Mallorca

Spain

Fax: 34971175228

E-mail: aagusti@hsd.es

Received

January 282007

Accepted after revision:

November 212007

\section{SUPPORT STATEMENT}

This work was supported by Fondo de Investigación Sanitaria (FIS 02/ 0986 and Red Respira-RTIC 03/11C; Spain), Sociedad Española de Neumología y Cirugía Torácica (SEPAR; Spain), Govern Balear (IIles Balears, Spain) and Associació Balear per l'Estudi de les Malalties Respiratòries (ABEMAR; IIles Balears, Spain)

STATEMENT OF INTEREST None declared. 
attention over the past few years [7-9]. This hypothesis is supported by several lines of evidence, particularly the fact that the inflammatory response previously alluded to persists after smoking cessation [4, 10-12], suggesting a self-perpetuating mechanism $[9,13]$ similar to those occurring in autoimmune diseases [7-9]. The hypothesis is further supported by recent data showing the presence of CD4+ T-lymphocyte oligoclonality in the lungs of patients with severe emphysema [14]; the possibility of inducing autoimmune emphysema in experimental animals [15]; and the very recent demonstration of circulating antibodies against elastin in patients with emphysema [16].

A small subpopulation of CD4+CD25+ T-lymphocytes, also known as regulatory T-cells (Tregs), with significant antiinflammatory and immunomodulatory effects, has been identified recently [17, 18]. Abnormalities in Treg regulation have been described in many chronic inflammatory and autoimmune disorders, including atherosclerosis and rheumatoid arthritis [17-20], but their involvement in COPD is unclear.

The present study sought to provide further evidence for the involvement of an acquired immune response in the pathogenesis of COPD by investigating: 1) maturation (CD45RA, CD45R0) and activation (CD28) markers of CD4+ and CD8+ T-lymphocytes (both cell types are believed to play a key role in the pathogenesis of COPD [3]); and 2) the presence of potential differences in the distribution of CD4+CD25+ Tregs in smokers with and without COPD, as well as in never-smokers.

\section{METHODS}

\section{Population and ethics}

The present study involved 23 patients with COPD (Global Initiative for Chronic Obstructive Lung Disease stage II-III), 29 smokers with normal lung function and seven never-smokers. All participants required bronchoscopy for the clinical evaluation of a solitary pulmonary nodule or haemoptysis, and all of them gave informed consent after being made fully aware of the nature and objectives of the study, which had been previously approved by the Ethics Committee of the present authors' institution (Comitè Ètic d'Investigació Clínica, Illes Balears, Spain).

COPD patients were clinically stable and had not had an exacerbation episode during the previous 3 months. All of them were being treated with long-acting inhaled bronchodilators and six patients also received inhaled steroids, but none was undergoing oral steroid therapy. Subjects with atopic diseases, allergic rhinitis and asthma were excluded. To avoid any potential effect of acute smoking, active smokers refrained from smoking $12 \mathrm{~h}$ before bronchoscopy. Exhaled carbon monoxide concentration was measured before the procedure and was $<10$ ppm in all subjects.

\section{Lung function}

Forced spirometry (GS; Warren E. Collins, Braintree, MA, USA) was obtained in all participants according to international guidelines [21]. Spirometric reference values were those of a Mediterranean population [22].

\section{Bronchoalveolar lavage fluid and blood samples}

Bronchoalveolar lavage was performed as previously reported in the current authors' laboratory [5,6]. Briefly, bronchoscopy was performed with a flexible fibreoptic bronchoscope (Pentax 15v; Pentax, Tokyo, Japan) under topical lidocaine. Eight 25$\mathrm{mL}$ aliquots of sterile saline solution were instilled in one pulmonary segment of a lower lobe without any lung nodule. The liquid recovered was filtered, washed twice in PBS and resuspended at $10^{6}$ cells $\cdot \mathrm{mL}^{-1}$ in RPMI-1640 medium at $4^{\circ} \mathrm{C}$. Blood samples were collected before bronchoscopy by peripheral venipuncture. Both bronchoalveolar lavage fluid (BALF) and peripheral blood samples were processed immediately after attainment.

\section{Flow cytometry analysis}

In order to characterise T-cells, blood and BALF samples were incubated with combinations of CD3, CD4, CD8, CD45RA, CD45R0 (Pharmingen, Becton Dickinson, Madrid, Spain), CD28, CD25, CD27 (Coulter Immunotech, Izasa, Spain), CD62L and glucocorticoid-induced tumour necrosis factor receptor (GITR; eBioscience, Bionova, Madrid, Spain) monoclonal antibodies for $20 \mathrm{~min}$ at room temperature in the dark. Subsequently, $2 \mathrm{~mL}$ of lysing solution was added and cells were washed and resuspended in PBS.

For intracellular detection of cytotoxic T-lymophocyteassociated antigen (CTLA)4, cells were fixed, permeabilised and stained with anti-CTLA4 (Coulter Immunotech) using the Intrastain Fixation and Permeabilization Kit (Dako, Glostrup, Denmark), according to the manufacturer's instructions.

Intracellular detection of Foxp3 was carried out using the antihuman Foxp3 (clone PCH101; eBioscience, Bionova) monoclonal antibody according to the manufacturer's instructions.

Fluorescence-activated cell sorting analyses were performed on an Epics XL flow cytometer using the Expo32 software (Coulter Immunotech).

\section{Statistical analysis}

Results are shown as mean \pm SEM. One-way ANOVA with post hoc Bonferroni contrasts was used to assess the statistical significance of differences between the three groups of subjects. An unpaired t-test was used to evaluate differences between BALF and blood sample parameters. A p-value $<0.05$ was considered significant.

\section{RESULTS}

\section{General findings}

Table 1 shows the main clinical and functional characteristics of participants. Age was similar in the three groups. The smoking history of patients with COPD was slightly higher than that of smokers with normal lung function. Patients with COPD showed moderate airflow obstruction, whereas spirometry was normal in the other two groups.

Table 2 shows the absolute and differential cell counts in BALF. BALF recovery was reduced in COPD; in contrast, total cell count was higher in COPD and in smokers with normal lung function.

\section{Phenotypic characterisation of CD4+ and CD8+ T-lymphocytes in blood and BALF}

In peripheral blood, the percentage of naïve (CD45RA+) and memory (CD45R0+) CD4+ and CD8+ T-lymphocytes was not significantly different between groups (table 3 ). 
TABLE 1 Clinical and functional data of all participants

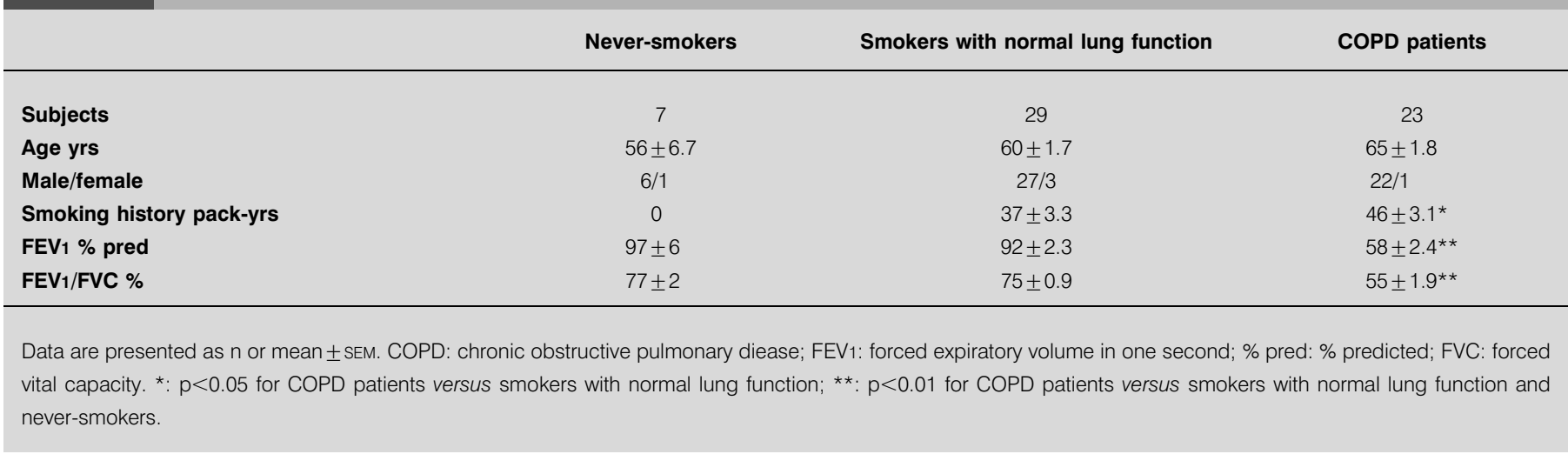

Compared with blood measurements, the percentage of memory (CD45R0+) CD4+ and CD8+ T-cells was significantly increased in BALF in all three groups. Conversely, naïve (CD45RA+) CD4+ and CD8+ T-lymphocytes were found in a higher proportion in blood than in BALF (table 3).

In BALF, the percentage of naïve and memory CD4+ T-cells was similar in all groups (table 3). In contrast, CD8+ T-lymphocytes showed significant differences between groups, with COPD patients showing higher CD8+ CD45RA+ and lower CD45R0+ percentages than smokers with normal lung function $(\mathrm{p}<0.001$ and $\mathrm{p}<0.05$, respectively).

Table 4 presents the expression of the co-stimulatory molecule CD28 in CD4+ and CD8+ T-lymphocytes, both in blood and BALF. In blood, values were not different between groups, neither for CD4+ nor CD8+ T-cells. In contrast, in BALF, irrespective of the presence of COPD, CD8+ T-cells from smokers have a lower percentage of CD8+ T-cells expressing CD28 than never-smokers (table 4).

\section{CD4+CD25+ Tregs}

Figure 1 presents the percentage of CD4+CD25+ in blood and BALF in the three groups of subjects studied. No significant differences in peripheral blood samples were found between groups. In contrast, it was observed that compared with neversmokers, smokers with preserved lung function showed a prominent upregulation of Tregs in BALF. Interestingly, this response was absent in smokers with COPD. It was also found that CD4+CD25+ were higher in BALF than in peripheral blood in the three groups of individuals studied (fig. 1).

Tregs were identified as CD4+ T-lymphocytes expressing bright CD25 (fig. 2), as well as other surface and intracytoplasmatic markers that characterise these cells. Therefore, CD4+CD25+ cells expressed higher levels of CTLA4/CD152, GITR, CD62L and CD27 than CD4+CD25- T-cells (fig. 3). They also expressed the memory surface marker CD45R0 and were negative for the activator marker CD69 (data not shown).

A characteristic of CD4+CD25+ T-cells is the production of interleukin (IL)-10, so intracellular IL-10 was explored in stimulated T-cells. It was found that a higher percentage of CD4+CD25+ T-cells stained positive for IL-10 than did CD4+CD25- ( $4.4 \pm 0.23 \%$ versus $0.1 \pm 0.06 \% ; \mathrm{p}<0.001)$.

Foxp3 is a forkhead transcription factor that is needed for the differentiation of Tregs and is a hallmark of CD4+CD25+ Tregs [23]. This led to the evaluation of the expression of intracellular Foxp3 in CD4+CD25+ T-cells. It was found that CD4+CD25+ T-cells, both in blood and BALF, expressed intracellular Foxp3 (fig. 4).

\section{DISCUSSION}

The present study sought first to characterise the maturationactivation phenotype of T-lymphocytes in patients with COPD, and secondly, to explore potential abnormalities in CD4+ CD25+ Tregs. Its main findings were that in BALF: 1) patients with COPD showed higher CD8+CD45RA+ $(p<0.001)$ and

TABLE 2 Absolute and differential cell counts in bronchoalveolar lavage fluid (BALF)

\begin{tabular}{lccc} 
& Never-smokers & Smokers with normal lung function & COPD patients \\
\hline Subjects & 7 & 29 & 23 \\
Total cell count $\times \mathbf{1 0}^{\mathbf{3}} \cdot \mathbf{m L}^{-1}$ & $63.8 \pm 15.3$ & $283 \pm 49^{\star}$ & $186 \pm 28$ \\
Macrophages \% & $93 \pm 1$ & $93 \pm 2$ & $86 \pm 2$ \\
Lymphocytes \% & $6 \pm 1$ & $6 \pm 1$ & $10 \pm 2$ \\
Neutrophils \% & $1 \pm 0.3$ & $1 \pm 0.2$ & $4 \pm 2$ \\
BALF recovery $\mathbf{~ L L}$ & $104 \pm 6$ & $95 \pm 3^{\#}$ & $69 \pm 5^{\star *}$ \\
\hline
\end{tabular}

Data are presented as $n$ or mean \pm SEM. COPD: chronic obstructive pulmonary disease. ${ }^{*}: p<0.05$ versus never-smokers; ${ }^{* *}: p<0.01$ versus never-smokers; ${ }^{\#}: p<0.01$ versus COPD patients. 


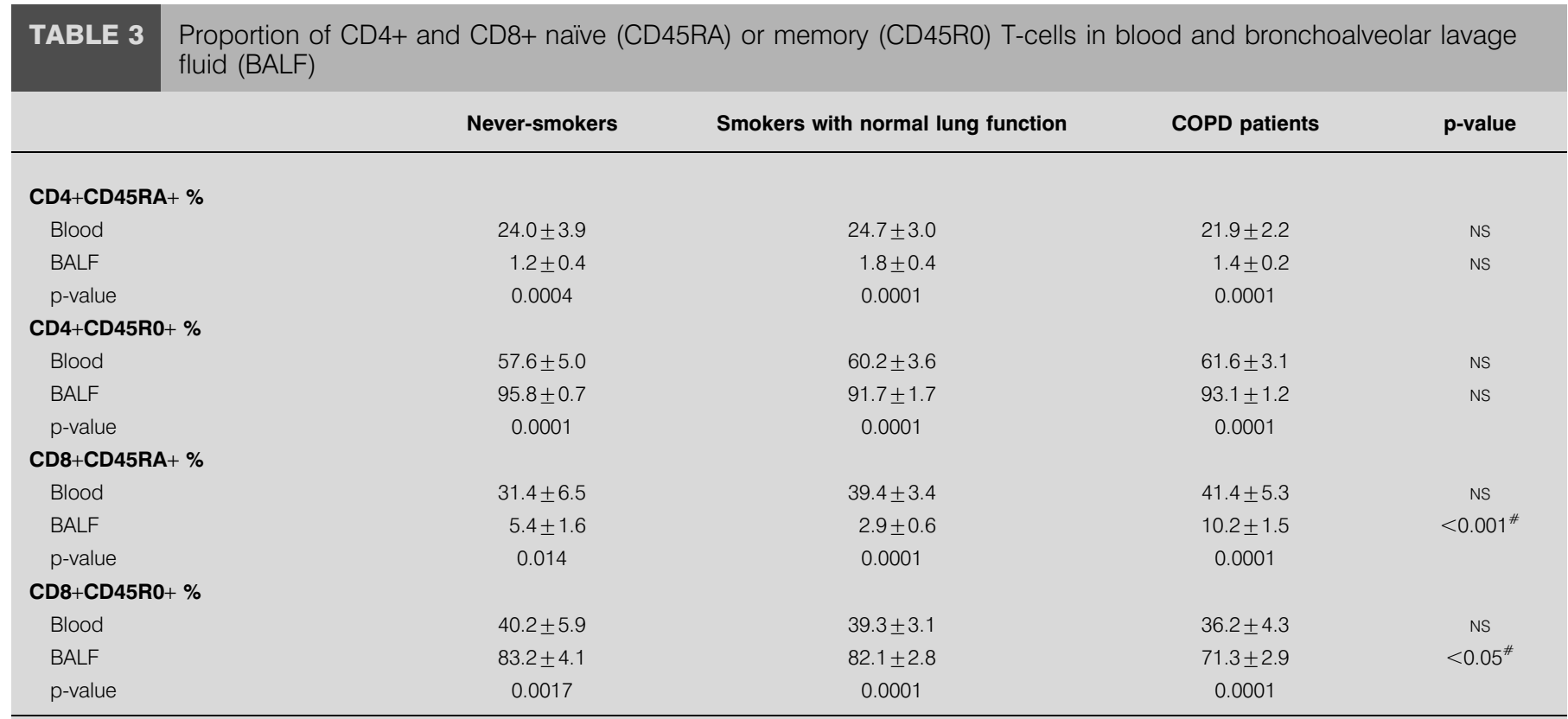

Data are presented as mean \pm SEM, unless otherwise stated. Percentage of CD4+CD45RA+/45R0+ or CD8+CD45RA $+/ 45 R 0+$ refers to the total amount of CD4 or CD8 T-cells, respectively. COPD: chronic obstructive pulmonary disease; ns: nonsignificant. \#: for COPD patients versus smokers.

lower CD8+CD45R0+ $(\mathrm{p}<0.005)$ than smokers with normal lung function (table 2); and 2) compared with never-smokers, smokers with preserved lung function showed a prominent upregulation of Tregs that was absent in patients with COPD (fig. 1). In addition, the present study provided two further observations of interest: the proportion of CD8+CD28+ was lower in smokers than in never-smokers, irrespective of the presence or absence of COPD; and in peripheral blood there were no significant differences between groups in any of the different markers studied, whereas their distribution varied markedly between blood and BALF. This suggests an active but physiological (it also occurred in never-smokers) compartmentalisation process of T-lymphocyte traffic.

Many previous studies have shown that T-lymphocytes accumulate in the lungs of patients with $\operatorname{COPD}[4,7,24-26]$ but, to the current authors' knowledge, only one has attempted to characterise the maturation-activation phenotype of T-lymphocytes in patients with COPD [14]. Sullivan et al. [14] reported a higher percentage of CD4+ and CD8+ T-cells expressing CD45R0 and a lower proportion expressing CD28 in the lung of patients with emphysema, as compared with in their own blood. This observation, which fully agrees with the present results (tables 3 and 4), indicates that the majority of these cells are mature/activated T-cells. However, whereas SulLIVAN et al. [14] suggested that this was indicative of activated effector memory T-cells being actively recruited into the lungs of patients with emphysema, the current authors' interpretation of this same finding is different because, at variance with the previous investigation, control subjects were also studied. By doing so, the present study found that the higher proportion of CD4+CD45R0+ and CD8+CD45R0+

\section{TABLE 4 Expression of the co-stimulatory molecule CD28 in CD4+ and CD8+ T-lymphocytes in blood and bronchoalveolar lavage fluid (BALF)}

\begin{tabular}{lccc} 
& Never-smokers & Smokers with normal lung function & COPD patients \\
\hline CD4+CD28+ \% & & & \\
Blood & $95.6 \pm 1.7$ & $98.4 \pm 0.7$ & $97.3 \pm 0.9$ \\
BALF & $58.0 \pm 11.2$ & $75.0 \pm 6.9$ & $71.1 \pm 5.3$ \\
P-value & 0.007 & 0.006 & 0.0001 \\
CD8+CD28+\% & & & 0.08 \\
Blood & $52.5 \pm 4.6$ & $55.2 \pm 8.6$ & $58.9 \pm 4.7$ \\
BALF & $57.6 \pm 6.3$ & $26.6 \pm 7.3$ & $29.1 \pm 4.7$ \\
P-value & NS & 0.026 & 0.0002 \\
\hline
\end{tabular}

Data are presented as mean $\pm \mathrm{SEM}$, unless otherwise stated. Percentages of $\mathrm{CD} 4+\mathrm{CD} 28+$ or $\mathrm{CD} 8+\mathrm{CD} 28+$ refer to the total amount of $\mathrm{CD} 4$ or $\mathrm{CD} 8 \mathrm{~T}$-cells respectively COPD: chronic obstructive pulmonary disease; NS: nonsignificant. ${ }^{\#}$ : for never-smokers versus smokers, with or without COPD. 


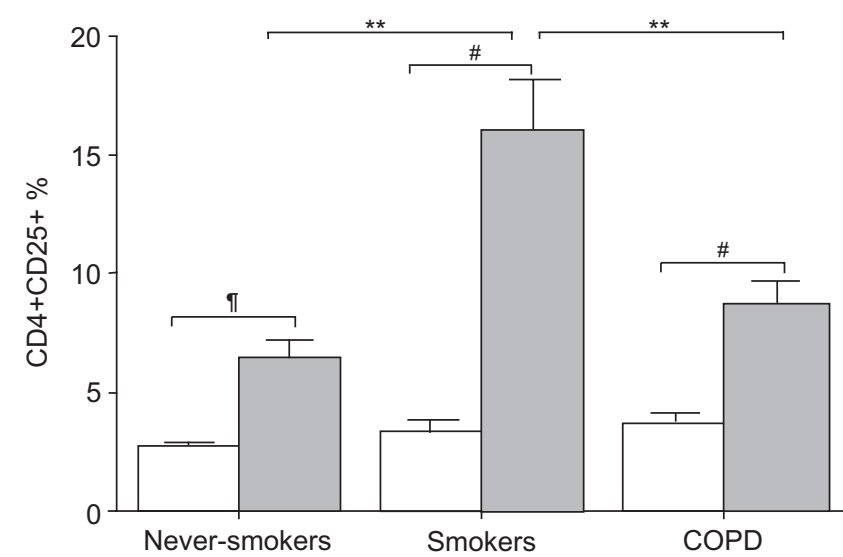

FIGURE 1. Percentage of $C D 4+C D 25+$ referred to the total amount of $C D 4+$ T-lymphocytes on peripheral blood ( $\square$ ) and bronchoalveolar lavage fluid ( $\square$ ) samples of never-smokers, smokers with normal lung function and chronic obstructive pulmonary disease (COPD) patients. ${ }^{*}: p<0.01 ;{ }^{*}: p<0.0001 ; ": p=0.0004$.

T-cells (as well as the lower percentage of $\mathrm{CD} 4+\mathrm{CD} 28+$ or $\mathrm{CD} 8+\mathrm{CD} 28+)$ reported in COPD also occurred in smokers with normal lung function and even in never-smokers (tables 3 and 4). Therefore, these changes cannot be directly linked to the pathogenesis of COPD and probably represent the physiological homing of mature T-cells in the lungs as compared with the general pool of circulating T-lymphocytes. Furthermore, the inclusion of controls allowed the present study to compare BALF data between groups and to unravel some interesting differences. First, it was observed that patients with COPD showed higher CD8+CD45RA+ and lower CD8+CD45R0+ levels than smokers with normal lung function (table 3). The normal maturation-activation process of T-lymphocytes involves the sequential expression of CD45RA (naïve T-cells), CD45R0 (mature T-cells) and, again, CD45RA (effector/cytotoxic T-cells) [27]. Therefore, the observation of a higher proportion of CD8+CD45RA+ T-lymphocytes in patients with COPD may indicate a final maturation-activation state of these cells (CD8+ cells expressing CD45RA and perforin) with a correspondingly higher potential for tissue injury [27]. However, the exact phenotype of these lymphocytes should be addressed in order to confirm the data. In contrast, the current authors observed that the percentage of CD4+ T-lymphocytes expressing the maturation markers CD45RA or CD45R0 in BALF was not significantly different between groups (table 3), suggesting a different role for these T-cells in the pathogenesis of the disease, such as the modulation of the immune response by CD4+CD25+ Tregs discussed hereafter.

Tregs constitute a small subpopulation of CD4+ T-lymphocytes expressing CD25, which have been recently identified as key immunomodulators in many chronic inflammatory and autoimmune disorders, including atherosclerosis and rheumatoid arthritis [17-20]. To the current authors' knowledge, the potential involvement of Tregs in COPD has not been explored before. This may be relevant because the involvement of an acquired immune response has been recently postulated as part of the pathogenesis of the disease $[4,7,8,14,15]$. Tregs maintain the homeostasis of the immune system, avoiding the

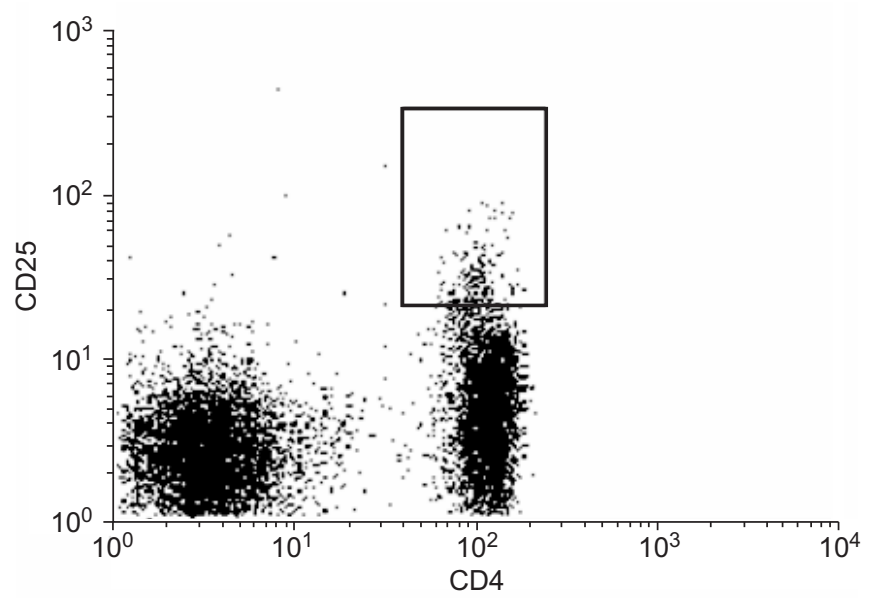

FIGURE 2. Regulatory T-cells were identified as CD4+CD25+bright (boxed area). Lymphocytes were gated on a forward versus side scatter dot plot. Percentages of CD4+CD25+ T-cells refer to the total amount of CD4+ T-cells.

activation of undesirable responses to self- and nonselfantigens. After activation, Tregs suppress proliferation of $\mathrm{CD} 4+$ and CD8+ T-cells through cell contact-dependent mechanisms and secretion of cytokines, mostly IL-10. The present study found that those smokers who manage to preserve their lung function despite their habit showed a prominent upregulation of Tregs in BALF compared with never-smokers, whereas this response was blunted in smokers who had developed COPD (fig. 1). Given the array of immunoregulatory functions of Tregs [17-20], their upregulation in smokers with normal lung function may be interpreted as an attempt to regulate and minimise the inflammatory response elicited by tobacco smoking, whereas the failure of this mechanism in patients with COPD may contribute to the enhancement and/or disregulation of such an inflammatory response, thus contributing to the pathogenesis of the disease. This pattern of response is, in fact, very similar to that of $\gamma \delta$ T-lymphocytes, another subpopulation of T-lymphocytes involved in tissue repair recently described by the current authors' group [6], and is in keeping with a recent report published while the present study was under review [16]. In any case, the abnormal response of Tregs in patients with COPD described here provides further support to the hypothesis that autoimmunity may play an important pathogenic role in COPD.

The present study provided two other observations of interest. First, smoking reduced the expression of the co-stimulatory molecule CD28 in CD8+ T-lymphocytes in BALF (but not in blood) and this occurred irrespectively of the presence or absence of COPD (table 4). This observation agrees with a report by EKBERG-JANSSON et al. [28], who also found lower expression of CD28 on CD8+ T-lymphocytes in BALF from smokers with normal lung function compared with neversmokers [28]. Given that the maturation process of T-lymphocytes involves the downregulation of CD28 [27], these observations suggest that mature $\mathrm{CD} 8+\mathrm{T}$-lymphocytes are being actively recruited into the lungs as a response to tobacco smoking that is not specifically linked to the pathogenesis of COPD. Secondly, no significant differences 
a)

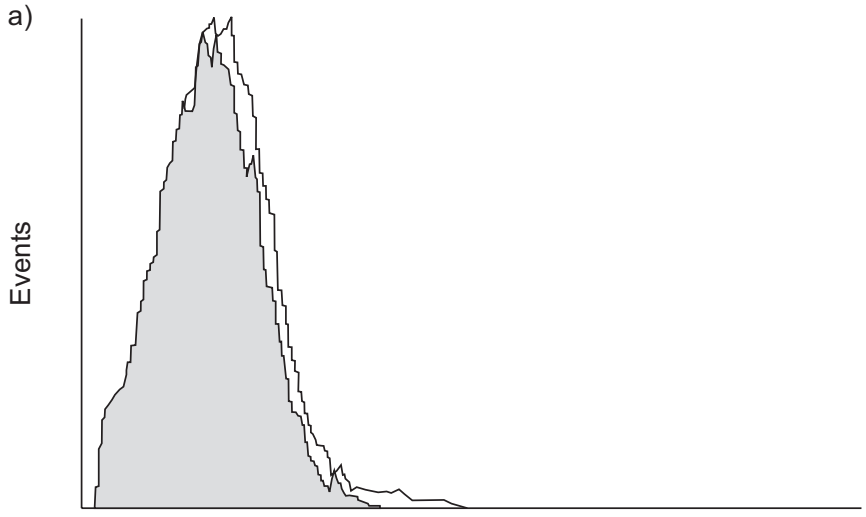

c)

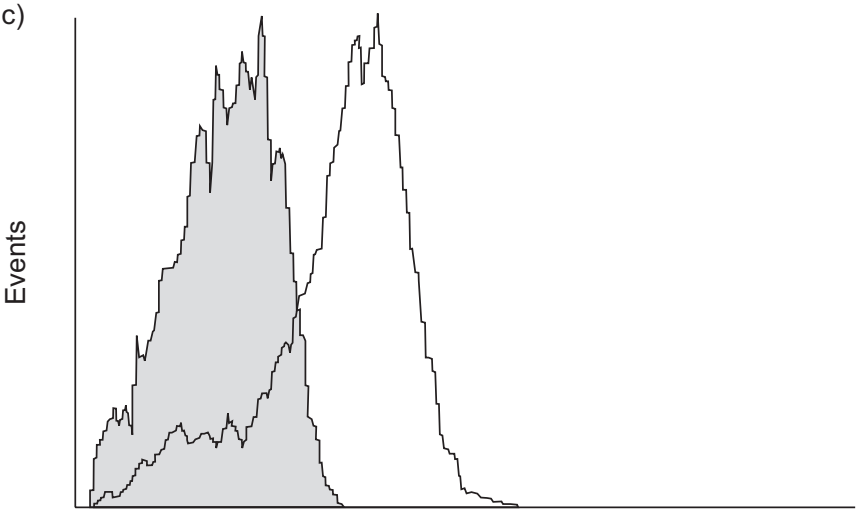

e)

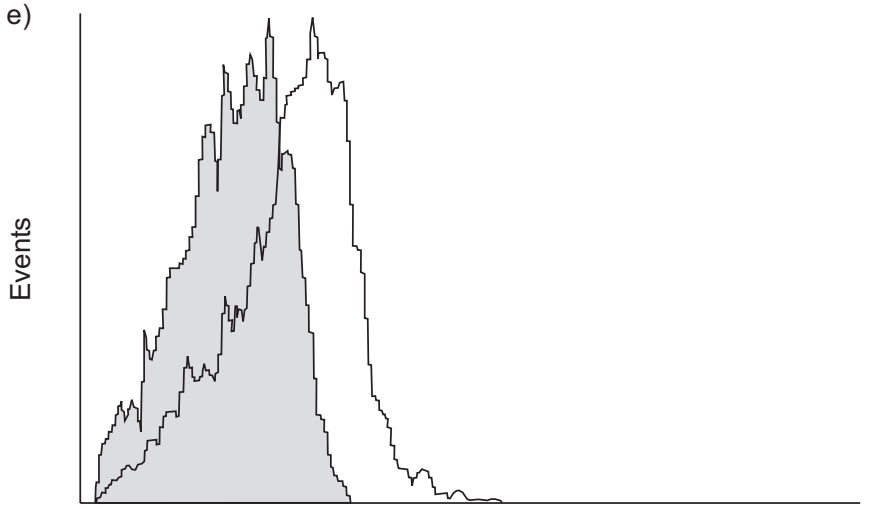

g)

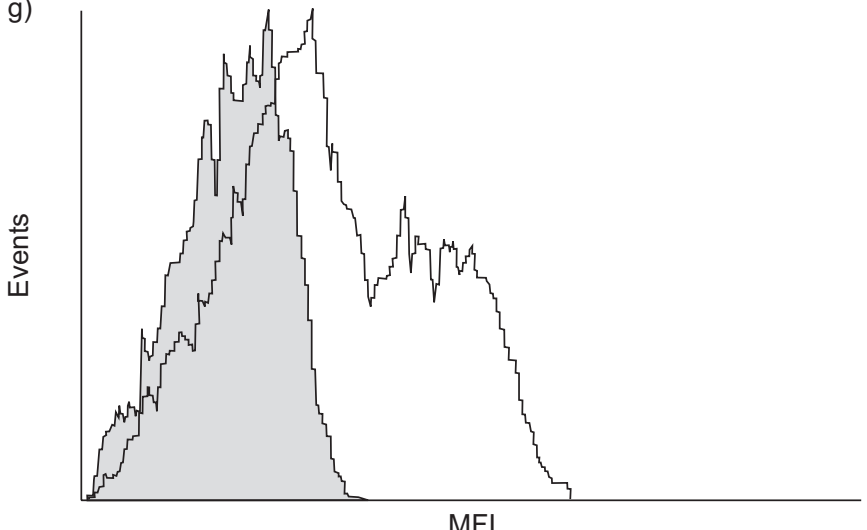

b)

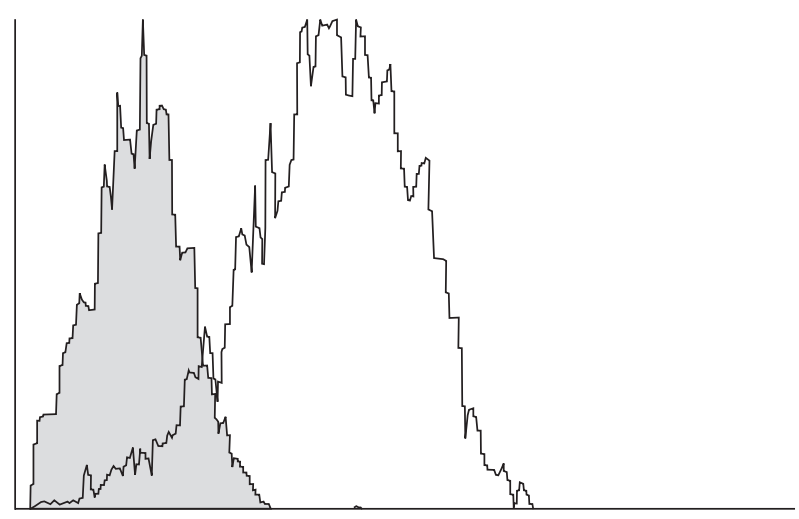

d)

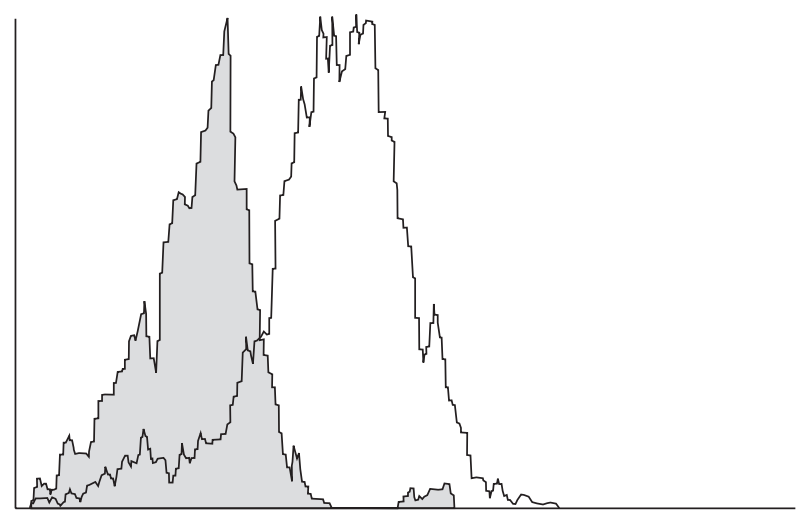

f)

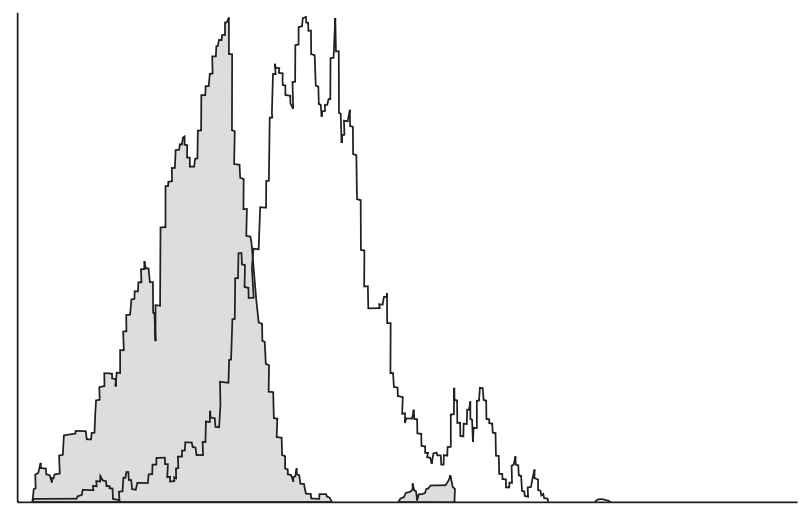

h)

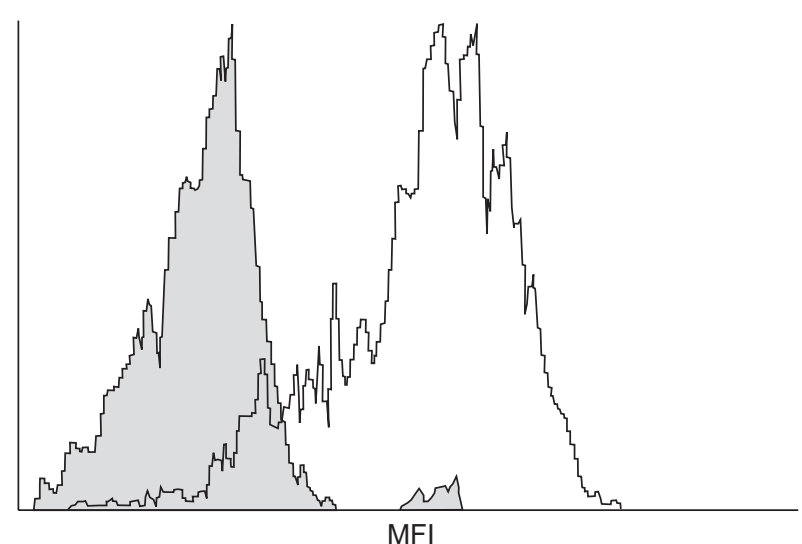

FIGURE 3. Flow cytometry analysis of cytotoxic T-lymphocyte-associated antigen (CTLA)4 (a, b), glucocorticoid-induced tumour necrosis factor receptor (GITR; c, d), CD62L (e, f) and CD27 ( $\mathrm{g}, \mathrm{h}$ ) expression. Representative histograms $(\square)$ of the mean fluorescence intensity (MFI) of cytoplasmic CTLA4 and surface GITR, CD62L and CD27 on bronchalveolar lavage fluid (BALF) CD4+CD25- ( $a, c$, e and g) and on BALF CD4+CD25+ (b, $d, f$ and h) T-cells. Mean \pm SEM of MFI values: a) 7.6 \pm 0.7 ; b) $39 \pm 3.1$; c) $13 \pm 5.2$; d) $33 \pm 7.3$; e) $8.8 \pm 2.3$; f) $30 \pm 4.4$; g) $20.7 \pm 6$; h) $92 \pm 18$. 1 : expression background level of cells stained with isotype-matched monoclonal antibodies. 

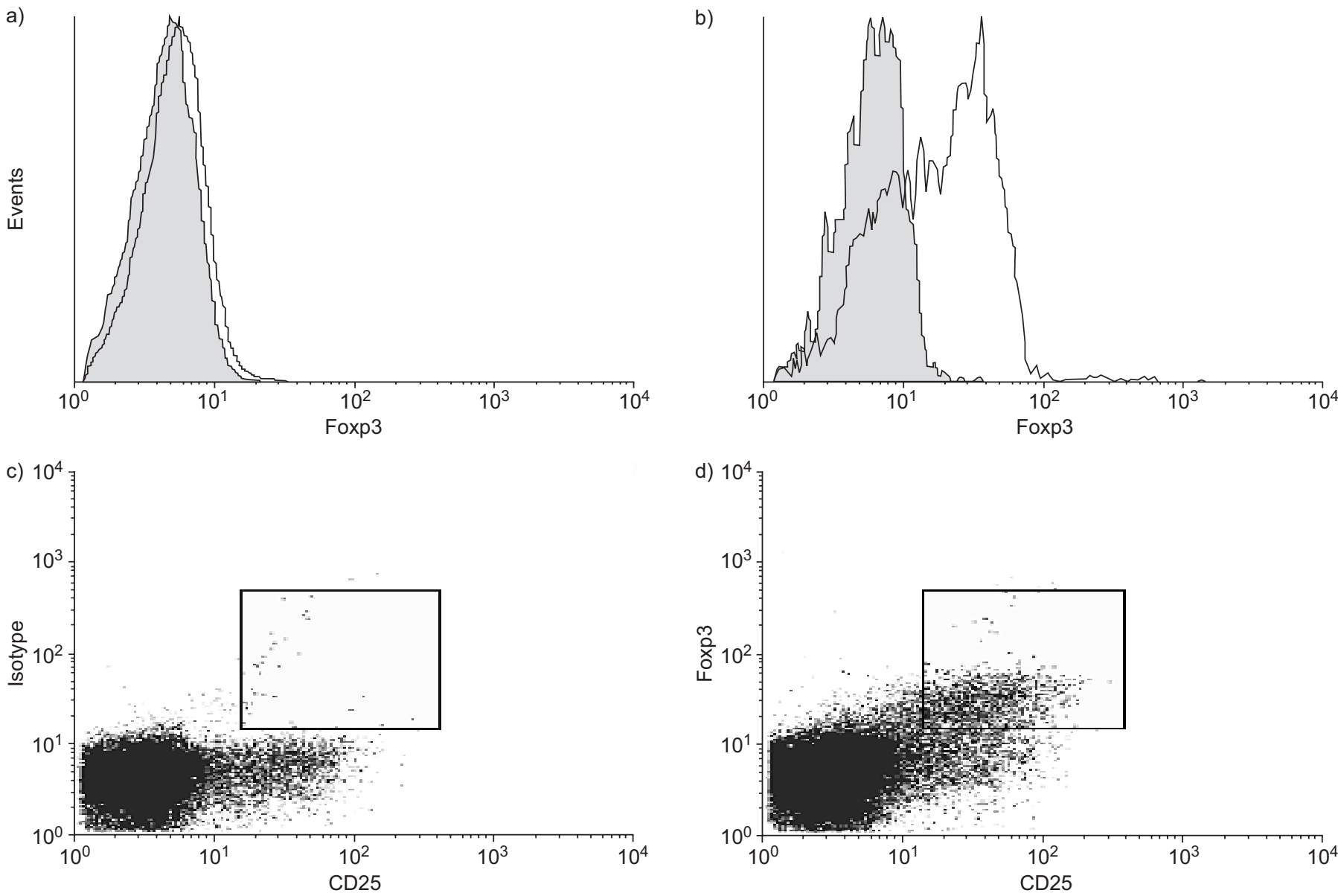

FIGURE 4. Flow cytometry analysis of Foxp3 (clone PCH101). Expression of cytoplasmic Foxp3 was evaluated on a) bronchoalveolar lavage fluid CD4+CD25- ( $\square$ ) and b) CD4+CD25+ T-cells $(\square)$. The mean \pm SEM values of Foxp3 expression were a) $5.8 \pm 0.85$ and b) $19 \pm 6.5$. $\square$ : expression background level of cells stained with isotypematched monoclonal antibodies. Representative dot plots of CD25 and c) immunoglobulin G isotype control (boxed area) and d) Foxp3 expression (boxed area) on CD4+ T-lymphocytes. Percentages, referred to the total amount of CD4+ T-cells, had values c) $0.09 \%$ and d) $2.06 \%$.

were found between groups in any of the different markers studied in peripheral blood, whereas marked compartmentalisation was observed when blood and BALF samples were compared (tables 3 and 4). These observations agree with previous studies [29-31] and suggest that differences observed in BALF (previously discussed) are due to specific recruitment and/or local activation in the lungs.

The present study has some limitations that deserve comment. First, BALF and blood samples were studied. Therefore, it is possible that T-lymphocytes isolated from other lung compartments (bronchial mucosa, interstitium) may show different phenotypic characteristics. Secondly, the population of CD8+ CD45RA+ T-lymphocytes isolated in BALF was not fully characterised in order to demonstrate that they represent the final stage of their maturation process. This should be done in future studies. Thirdly, six patients received inhaled steroids. For this reason, the results obtained in patients receiving or not receiving inhaled steroids were compared, and no significant differences were found. Fourthly, COPD patients had a longer history of cigarette smoking than smokers without COPD. Therefore, an effect of smoking cannot be excluded. However, the absolute difference in smoking exposure between the two groups (9 pack-yrs) was relatively small.

In summary, the present study is the first to characterise the maturation-activation phenotype of T-lymphocytes in patients with chronic obstructive pulmonary disease in comparison to that of smokers with normal lung function and never-smokers. It was found that patients with chronic obstructive pulmonary disease showed increased percentages of CD8+CD45RA+ T-lymphocytes, which is consistent with a final maturationactivation state of these cells with a correspondingly higher potential for tissue injury. Also, the present study is the first to investigate potential abnormalities in CD4+CD25+ regulatory $\mathrm{T}$-cells in these patients. It was found that the normal response of regulatory $\mathrm{T}$-cells to tobacco smoking is blunted in patients with chronic obstructive pulmonary disease, further supporting a potential involvement of the acquired immune response in the pathogenesis of chronic obstructive pulmonary disease.

\section{ACKNOWLEDGEMENTS}

The authors thank the participants of the study for their willingness to contribute to medical research, as well as 
F. Bauzá, A. Noguera, M. Bosch and C. Rendón (all Hospital Universitari Son Dureta, Palma de Mallorca, Spain) for their help during the study.

\section{REFERENCES}

1 Celli BR, MacNee W, Agusti AG, et al. Standards for the diagnosis and treatment of patients with COPD: a summary of the ATS/ERS position paper. Eur Respir J 2004; 23: 932-946.

2 Fletcher C, Peto R. The natural history of chronic airflow obstruction. BMJ 1977; 1: 1645-1648.

3 Hogg JC. Pathophysiology of airflow limitation in chronic obstructive pulmonary disease. Lancet 2004; 364: 709-721.

4 Hogg JC, Chu F, Utokaparch S, et al. The nature of smallairway obstruction in chronic obstructive pulmonary disease. N Engl J Med 2004; 350: 2645-2653.

5 Pons AR, Noguera A, Blanquer D, Sauleda J, Pons J, Agusti AGN. Phenotypic characterisation of alveolar macrophages and peripheral blood monocytes in COPD. Eur Respir J 2005; 25: 647-652.

6 Pons J, Sauleda J, Ferrer JM, et al. Blunted $\gamma \delta$ T-lymphocyte response in chronic obstructive pulmonary disease. Eur Respir J 2005; 25: 441-446.

7 Majo J, Ghezzo H, Cosio MG. Lymphocyte population and apoptosis in the lungs of smokers and their relation to emphysema. Eur Respir J 2001; 17: 946-953.

8 Agusti A, MacNee W, Donaldson K, Cosio M. Hypothesis: does COPD have an autoimmune component? Thorax 2003; 58: 832-834.

9 Barnes PJ, Cosio MG. Characterization of T lymphocytes in chronic obstructive pulmonary disease. PLoS Med 2004; 1: e20.

10 Turato G, Di Stefano A, Maestrelli P, et al. Effect of smoking cessation on airway inflammation in chronic bronchitis. Am J Respir Crit Care Med 1995; 152: 1262-1267.

11 Willemse BWM, ten Hacken NHT, Rutgers B, LesmanLeegte IGAT, Postma DS, Timens W. Effect of 1-year smoking cessation on airway inflammation in COPD and asymptomatic smokers. Eur Respir J 2005; 26: 835-845.

12 Retamales I, Elliot MW, Meshi B, et al. Amplification of inflammation in emphysema and its association with latent adenoviral infection. Am J Respir Crit Care Med 2001; 164: 469-473.

13 Grumelli S, Corry DB, Song LZ, et al. An immune basis for lung parenchymal destruction in chronic obstructive pulmonary disease and emphysema. PLoS Med 2004; 1: e8.

14 Sullivan AK, Simonian PL, Falta MT, et al. Oligoclonal CD4+ $\mathrm{T}$ cells in the lungs of patients with severe emphysema. Am J Respir Crit Care Med 2005; 172: 590-596.

15 Taraseviciene-Stewart L, Scerbavicius $\mathrm{R}, \mathrm{Choe} \mathrm{KH}$, et al. An animal model of autoimmune emphysema. Am J Respir Crit Care Med 2005; 171: 734-742.

16 Lee SH, Goswami S, Grudo A, et al. Antielastin autoimmunity in tobacco smoking-induced emphysema. Nat Med 2007; 13: 567-569.
17 Bluestone JA, Tang Q. How do CD4+CD25+ regulatory T cells control autoimmunity? Curr Opin Immunol 2005; 17: 638-642.

18 Jiang H, Chess L. Regulation of immune responses by $\mathrm{T}$ cells. N Engl J Med 2006; 354: 1166-1176.

19 Mottonen M, Heikkinen J, Mustonen L, Isomaki P, Luukkainen R, Lassila O. CD4+ CD25+ T cells with the phenotypic and functional characteristics of regulatory $\mathrm{T}$ cells are enriched in the synovial fluid of patients with rheumatoid arthritis. Clin Exp Immunol 2005; 140 360-367.

20 Ait-Oufella H, Salomon BL, Potteaux S, et al. Natural regulatory $\mathrm{T}$ cells control the development of atherosclerosis in mice. Nat Med 2006; 12: 178-180.

21 Standardization of spirometry, 1994 update. American Thoracic Society. Am J Respir Crit Care Med 1995; 152: 1107-1136.

22 Roca J, Sanchis J, Agustí-Vidal A, et al. Spirometric reference values for a Mediterranean population. Bull Eur Physiopathol Respir 1986; 22: 217-224.

23 Ahmadzadeh M, Rosenberg SA. IL-2 administration increases CD4+CD25 ${ }^{\text {hi }}$ Foxp3+ regulatory $\mathrm{T}$ cells in cancer patients. Blood 2006; 107: 2409-2414.

24 Finkelstein R, Fraser RS, Ghezzo H, Cosio MG. Alveolar inflammation and its relation to emphysema in smokers. Am J Respir Crit Care Med 1995; 152: 1666-1672.

25 Saetta M, Di Stefano A, Turato G, et al. CD8+ Tlymphocytes in peripheral airways of smokers with chronic obstructive pulmonary disease. Am J Respir Crit Care Med 1998; 157: 822-826.

26 Saetta M, Baraldo S, Corbino L, et al. CD8+ve cells in the lungs of smokers with chronic obstructive pulmonary disease. Am J Respir Crit Care Med 1999; 160: 711-717.

27 Sallusto F, Geginat J, Lanzavecchia A. Central memory and effector memory $\mathrm{T}$ cell subsets: function, generation, and maintenance. Annu Rev Immunol 2004; 22: 745-763.

28 Ekberg-Jansson A, Andersson B, Arva E, Nilsson O, Lofdahl CG. The expression of lymphocyte surface antigens in bronchial biopsies, bronchoalveolar lavage cells and blood cells in healthy smoking and never-smoking men, 60 years old. Respir Med 2000; 94: 264-272.

29 Costabel U, Bross KJ, Reuter C, Rühle K, Matthys H. Alterations in immunoregulatory $\mathrm{T}$-cell subsets in cigarette smokers. A phenotypic analysis of bronchoalveolar and blood lymphocytes. Chest 1986; 89: 39-44.

30 Ekberg-Jansson A, Arva E, Nilsson O, Lofdahl CG, Andersson B. A comparison of the expression of lymphocyte activation markers in blood, bronchial biopsies and bronchoalveolar lavage: evidence for an enrichment of activated $\mathrm{T}$ lymphocytes in the bronchoalveolar space. Respir Med 1999; 93: 563-570.

31 Korn S, Wiewrodt R, Walz YC, et al. Characterization of the interstitial lung and peripheral blood $\mathrm{T}$ cell receptor repertoire in cigarette smokers. Am J Respir Cell Mol Biol 2005; 32: 142-148. 